\title{
INSTRUMENTO DE AVALIAÇÃO MULTIDIMENSIONAL DA PESSOA IDOSA COM BASE NO MODELO DA CIF
}

Multidimensional assessment tool for elderly ICF based-on

Emilene Nóbrega Medeiros, $\quad$ emilenenobrega@gamil.com,
http://lattes.cnpq.br/1840016928796632, Psicóloga e Doutora em Enfermagem pelo
PPGENF/UFPB, ORCID iD: https://orcid.org/0000-0002-9087-0629
Patrícia Josefa Fernandes Beserra, ticinhajfb@hotmail.com, ID Lattes: http://lattes.cnpq.br/9321331636159057, Enfermeira e Pós-Doutorado em Enfermagem pelo PPGENF/UFPB, ORCID iD: https://orcid.org/0000-0002-4190-8280

Maria Miriam Lima da Nóbrega, miriamnobrega@gmail.com, ID Lattes: http://lattes.cnpq.br/5234341422159267, Enfermeira e Doutora em Enfermagem pelo PPGENF/UFPB, Orcid iD: https://orcid.org/0000-0002-6431-0708

Contato principal:+55 $8398898-4680$

\section{RESUMO}

Objetivos: Construir um instrumento de avaliação multidimensional para a pessoa idosa, embasada na CIF, e realizar a sua validação de conteúdo e de aparência. Método: Pesquisa metodológica, desenvolvida em três fases 1. Identificação das categorias da CIF para a Avaliação Multidimensional da Pessoa Idosa; 2. Construção do Instrumento; 3 Validação de conteúdo e aparência do Instrumento construído. Resultados: $A$ identificação das categorias da CIF, na literatura e pelos profissionais de saúde, permitiu a construção do Instrumento para Avaliação Multidimensional da Pessoa Idosa, estruturado a partir dos componentes da CIF, o qual foi submetido à Validação de conteúdo e de aparência por especialistas. Conclusão: Acredita-se que instrumento concluído, embasado no modelo teórico da CIF, vai contribuir com o diagnóstico multidimensional e o plano de cuidados da equipe de saúde para a pessoa idosa, mas reconhecese a necessidade da continuação do estudo para o desenvolvimento da etapa de validação clínica.

Descritores: CIF. Idoso. Atenção Primária à Saúde.

\begin{abstract}
Objectives: Build a multidimensional assessment instrument for the elderly, based on the ICF, and carry out its content and appearance validation. Method: Methodological research, developed in three phases 1. Identification of the ICF categories for the Multidimensional Assessment of the Elderly; 2. Construction of the Instrument; 3 Validation of content and appearance of the built Instrument. Results: The identification of the ICF categories, in the literature and by health professionals, allowed the construction of the Instrument for Multidimensional Assessment of the Elderly Person, structured based on the ICF components, which was submitted to content and appearance validation by specialists. Conclusion: It is believed that the completed instrument, based on the theoretical model of the ICF, will contribute to the multidimensional diagnosis and care plan of the health team for the elderly, but the need for further study is recognized for the development of the clinical validation step.
\end{abstract}

Descriptors: ICF. Elderly. Primary Health Care. 


\section{INTRODUÇÃO}

A saúde da pessoa idosa requer conhecimentos específicos, ainda muito centrados nas especialidades de geriatria e gerontologia, e muitas dessas especificidades são decorrentes do envelhecimento ser um processo multidimensional. $O$ aumento dos riscos das multimorbidades, das interferências da história de vida de cada indivíduo, das influências ambientais, e de muitos outros fatores que intervêm nos estados de saúde nessa fase de vida, corroboram para a complexidade dessa etapa de vida.

Avaliar o estado de saúde do idoso utilizando, exclusivamente, estatísticas de mortalidade e morbidades, pode não fornecer um quadro detalhado e efetivo das reais condições de vida e saúde desse indivíduo. São necessários indicadores de morbidade que abordem as incapacidades, que descrevam 0 impacto da doença/incapacidade sobre a família, o sistema de saúde e a qualidade de vida dos idosos ${ }^{1}$.

Nesse contexto, a avaliação funcional seria a estratégia de maior alcance de informações que estão inseridas na vida do idoso, permitindo um planejamento assistencial mais adequado, verificando, de forma sistematizada, em que grau as doenças impedem a sua capacidade funcional, seu campo de conhecimento e da prática, que se constrói de forma cumulativa, necessitando muitas vezes de uma e mais escalas de avaliação da capacidade funcional ${ }^{2}$.

Discussões referentes à capacidade funcional, principalmente na população idosa, escalas, instrumentos de avaliação e protocolos assistenciais são necessários e devem ser instituídos desde os serviços primários até os serviços terciários de saúde, buscando focar a multidimensionalidade da saúde do idoso presente no processo de envelhecimento, naturalmente.

Portando, entende-se por avaliação multidimensional da pessoa idosa, um somatório de todas as informações possíveis do idoso e deve ser complementada sempre que for preciso; seu objetivo maior é reconhecer as incapacidades no que ser refere à independência e autonomia em todos os seus aspectos: a funcionalidade global, os sistemas funcionais principais, os sistemas fisiológicos principais, medicamentoso, fatores contextuais, avaliação sociofamiliar, avaliação de cuidadores e avaliação funcional ${ }^{3}$.

Como base para compreender toda a visão biopsicossocial que a saúde do idoso requer, deve-se relembrar que a CIF, [...] contempla nas definições, os aspectos físicos, ambientais e subjetivos do indivíduo e da sua saúde ao deixar claro que a funcionalidade abrange todas as funções e sistemas do corpo, atividades e participações do indivíduo tanto na perspectiva individual como social e os aspectos positivos do indivíduo na relação com fatores pessoais e ambientais ${ }^{4-p .82}$.

A CIF objetiva estabelecer uma linguagem padrão e uma estrutura que descreve a saúde e os estados relacionados à saúde, seus determinantes e feitos, por meio de uma base científica, bem como permitir comparação de dados entre países, entre disciplinas, entre serviços e em diferentes momentos ao longo do tempo e fornece um esquema de codificação para sistemas de informações em saúde. Por agrupar sistematicamente diferentes domínios da saúde e domínios do bem-estar à saúde, descritos com base na perspectiva do corpo, do indivíduo e da sociedade, a CIF é dividida em duas partes: as Funções e Estruturas do Corpo, e as Atividades e Participação. Esses termos foram substituídos pelos anteriormente utilizados: deficiência, incapacidade e limitação ${ }^{5}$.

Também considerada um dicionário de funcionalidade humana que contém itens relacionados a partes do corpo e seu funcionamento, itens sobre as atividades humanas, participação social, bem como itens que influenciam nessas atividades, denominados fatores ambientais. Devido a 
essa complexidade, dispõe as informações na primeira parte à Funcionalidade e à Incapacidade e na segunda parte referente aos Fatores contextuais ${ }^{6}$.

A CIF não classifica pessoas, mas descreve a condição de cada pessoa contextualizada em uma escala de domínios de saúde, colocando todas as condições de saúde em um mesmo nível, permitindo ser avaliada por meio de uma régua métrica comum, com foco na saúde e na incapacidade que passa a recebe um novo olhar ${ }^{7}$.

Esse novo modelo é visto como multidirecional, onde uma deficiência ou incapacidade pode ser gerada a partir de qualquer item mencionado, existindo uma correlação entre todos, sem linearidade, servindo assim como um guia clínico para atuação em Saúde Funcional. Baseia-se numa abordagem biopsicossocial utilizada para se obter a integração das várias perspectivas de funcionalidade, e considera o indivíduo e toda influência dos componentes que favorecem ou dificultam a execução das suas atividades ${ }^{7,5}$.

No modelo biopsicossocial, uma pessoa pode, por exemplo, ter deficiência sem limitações de capacidade ou ter problemas de desempenho e limitações de capacidade sem deficiências evidentes ou ter limitações de capacidade sem assistência e nenhum problema de desempenho no ambiente. Consequentemente, é importante coletar dados sobre os componentes de maneira independente e, em seguida, explorar as associações e ligações existentes, pois todos eles são úteis para descrever a experiência de saúde em sua totalidade para cada indivíduo ${ }^{5}$.

Seu objetivo maior é a elaboração de uma síntese que possa descrever uma visão coerente das diversas dimensões de saúde sob as perspectivas biológica, individual e social, perfazendo uma relação multidirecional, onde a doença pode ser tanto o ponto de partia como o resultado de alterações primárias nos outros domínios do modelo ${ }^{5,7}$.
Infere-se que o desenvolvimento e a utilização de um Instrumento para Avaliação Multidimensional da Pessoa Idosa, tendo como base a CIF, podem contribuir na definição do diagnóstico multidimensional e do plano de cuidados da equipe, bem como na melhoria da qualidade da prestação da assistência à pessoa idosa e aos seus familiares, com foco na APS por meio da ESF.

Este artigo tem como objetivos construir um instrumento de avaliação multidimensional para a pessoa idosa, a ser utilizado na Estratégia Saúde da Família, embasada pelo modelo da Classificação Internacional de Funcionalidade, Incapacidade e Saúde, visando o diagnóstico multidimensional e o plano de cuidados para equipe de saúde, e realizar a sua validação de conteúdo e aparência.

\section{Método}

Trata-se de um estudo metodológico, o qual propõe uma organização sistemática para elaboração, avaliação, validação e aperfeiçoamento de instrumentos e técnicas de pesquisa, assim como de estratégias metodológicas, comumente utilizando métodos complexos e sofisticados, incluindo métodos mistos ${ }^{8}$.

Antes da sua realização obteve a apreciação pelo Comitê de Ética em Pesquisa, do Centro de Ciências da Saúde (CCS) da Universidade Federal da Paraíba, levando em consideração os aspectos éticos preconizados pela Resolução No 466/12 do Conselho Nacional de Saúde, que regulamenta a pesquisa em seres humanos ${ }^{9}$, tendo sido aprovado segundo o CAAE $n$. 09355919.1.0000.5188. Durante a pesquisa foram esclarecidos os objetivos do estudo, a garantia do anonimato, os procedimentos para coleta de dados e assinatura do Termo de Consentimento Livre e Esclarecido (TCLE).

Para atingir os objetivos propostos 0 estudo foi desenvolvido em três etapas: 1. Identificação das categorias da CIF para a Avaliação Multidimensional da Pessoa Idosa, 
executada em dois momentos; 2. Construção do Instrumento para Avaliação Multidimensional da Pessoa Idosa; 3 Validação de conteúdo e aparência do Instrumento para a Avaliação Multidimensional da Pessoa Idosa - IAMPI. Essas etapas foram executadas, utilizando métodos e técnicas específicas para o alcance dos objetivos da pesquisa, as quais estão definidas e estruturadas a seguir.

\section{Identificação das categorias da CIF para a Avaliação Multidimensional da Pessoa Idosa}

$\mathrm{Na}$ construção do Instrumento para Avaliação Multidimensional da Pessoa Idosa IAMPI, inicialmente buscou-se 0 conhecimento técnico de avaliadores, tanto no conhecimento clínico e prático, como no conhecimento teórico, e sua afinidade com a $\mathrm{CIF}$, que foi utilizada como base teórica para sua estruturação.

Foram desenvolvidas nessa etapa duas fases: a primeira objetivou 0 desenvolvimento de uma revisão integrativa da literatura para identificar nos últimos 10 anos quais as categorias da CIF mais relevantes para 0 atendimento multiprofissional na avaliação funcional da pessoa idosa. A segunda fase, buscou a identificação das categorias da CIF que representam objetivos potenciais para 0 atendimento multiprofissional para a pessoa idosa, realizada, em dois momentos, ambos com a participação de um grupo de profissionais com experiência prática do atendimento multiprofissional na avaliação funcional da pessoa idosa.

Para tanto, foi elaborado um instrumento, utilizando o Google Forms $\AA$, contendo todos os componentes sinalizados com maior representatividade nos estudos identificados na primeira fase, tendo como base o checklist da OMS. O instrumento eletrônico foi construído com perguntas relacionadas, inicialmente, à caracterização dos profissionais participantes quanto as variáveis pessoais (sexo, idade, unidade federativa de residência, titulação máxima, área e tempo de atuação profissional) e temáticas (avaliação funcional da pessoa idosa e categorias da CIF); e um checklist das categorias principais da CIF contendo também os termos identificados da primeira fase deste estudo, dividido em Funções do Corpo (funções fisiológicos dos sistemas do corpo, incluindo funções psicológicas), Estruturas do Corpo (partes anatômicas do corpo como órgãos, membros e seus componentes), Atividade (execução de uma tarefa ou ação por um indivíduo), Participação (envolvimento em situação de vida diária), Fatores Ambientais (compõem o ambiente físico, social e de atitude no qual as pessoas vivem e conduzem sua vida), e Fatores Pessoais (histórico particular da vida e do estilo de vida de um indivíduo).

Os avaliadores do estudo receberam orientações quanto ao foco do instrumento, de mensurar as informações sobre a funcionalidade, capacidade, e a incapacidade de um indivíduo, as quais foram identificadas na literatura da área e no checklist da CIF. Como a sensibilização de que cada um poderia colaborar com novas sinalizações de componentes, dentro de cada categoria da $\mathrm{CIF}$, que fossem vistos como essenciais para uma avaliação multidimensional da saúde da pessoa idosa. Também foi solicitado o consentimento para participar da pesquisa, com a assinatura digital do TCLE.

A seleção dos participantes nessa fase foi feita a partir da base de dados da Plataforma Lattes, levando em consideração os critérios: profissionais de saúde com experiência prática no atendimento multiprofissional na avaliação funcional da pessoa idosa e que trabalhem na Estratégia Saúde da Família, dando preferência por estudiosos do assunto e produtores de artigos específicos sobre o tema em questão (CIF, Avaliação Multidimensional, Pessoa idosa, Atenção Primária à Saúde). Também foram selecionados profissionais da saúde que 
trabalhavam em serviços da estratégia saúde da família atendendo a clientela especificada em João Pessoa - PB, por meio de contato pessoal, ou via e-mail ou telefone, que preenchessem os critérios de inclusão. Após seleção dos especialistas encaminhou-se, via web, carta convite a 46 profissionais, que preencheram os critérios de inclusão e que residiam no Brasil. $\mathrm{Na}$ composição da amostra, solicitou-se que os especialistas selecionados indicassem outros profissionais da saúde que atendessem aos critérios estabelecidos para fazerem parte do estudo.

Nesta pesquisa, foi considerada uma amostra mínima de aproximadamente 15 especialistas, tendo como base a realização de estudos semelhantes, porém aceitaram participar do estudo 25, com a devolução dos instrumentos em outubro de 2019, perfazendo quatro meses de coleta. Para a análise dos dados gerados a partir do instrumento, foi utilizado o método de Índice de Validade de Conteúdo - IVC que mede a proporção ou porcentagem de especialistas que estão em concordância sobre determinados aspectos do instrumento e de seus itens ${ }^{10}$. Foram considerados validados os itens que obtiverem uma concordância de no mínimo IVC $\geq 0.80$.

Para dar continuidade ao estudo, foi necessário realizar a paridade entre as categorias da CIF identificadas na literatura e nas categorias da CIF apontadas como relevantes pelos profissionais da saúde, enumerados os componentes que seriam relevantes para compor o instrumento. Em seguida, foi configurado um novo instrumento utilizando o Google Forms ${ }^{\circledR}$, contendo as categorias e conceitos, com suas respectivas definições descritas na CIF, e encaminhada via web, em dezembro de 2019, para os 25 juízes que fizeram parte da etapa inicial para avaliar as similaridades de conteúdo. Foram devolvidos oito instrumentos no período de janeiro a março de 2020, perfazendo três meses de coleta. Vale ressaltar que nesse período começou a pandemia do coronavírus no Brasil, e acredita-se que este fato tenha contribuiu para a não participação de todos os juízes nessa segunda fase.

\section{Construção do Instrumento para Avaliação Multidimensional da Pessoa Idosa}

Seguindo o padrão dos demais instrumentos encontrados na literatura ${ }^{11}$, a primeira parte do IAMPI foi construído contendo os Fatores Pessoais, outros dados sociodemográficos, e algumas informações fundamentais para uma avaliação multidimensional, como medicações utilizadas e condições crônicas existentes. Dentre vários itens sinalizados na literatura como fatores sociodemográficos, os itens descritos nesse instrumento foram considerados relevantes tanto no âmbito teórico como na prática clínica. Foram descritos em seu arcabouço as informações, para que o avaliador obtivesse o maior número de dados necessárias e que sua visibilidade não fosse de algo extenso.

A segunda parte do IAMPI contém as categorias validadas com ICV $\geq 0.80$, seguindo a mesma estruturação da CIF, ou seja, Funções do Corpo, Estruturas do Corpo, Atividades e Participação e Fatores Ambientais. Os termos de cada um desses componente foram definidos de acordo com a CIF, a princípio, para uma organização e, consequentemente, em virtude do seu volume, para uma compreensão de sua totalidade e esclarecimento do seu entendimento segundo o modelo teórico a ser seguido. Cada termo recebeu uma leitura criteriosa e na integra, objetivando o contexto de cada um e assim proporcionar melhor elaboração das perguntas para, consequentemente, obter respostas com foco sempre na funcionalidade da pessoa idosa.

Concomitamente a essa etapa, os itens foram, mais uma vez, emparelhados entre si, com finalidade de identificar as semelhanças entre os termos, para que não ocorressem repetições de conteúdo, ou subvalorização 
com relação a outros itens. Em seguida cada item com IVC $\geq 0.80$, acompanhado de sua codificação alfanumérica, teve sua definição descrita, retirada da CIF. Após essa organização de informações, foi elaborada uma pergunta, tendo como base a definição proposta e exemplos de outros instrumentos, e posteriormente os qualificadores SIM ou NÃO, considerando o SIM, um indivíduo com funcionalidade positiva.

Posteriormente, a elaboração das perguntas referente ao componente Funções do corpo buscou manter a essência de sua definição, ao mesmo tempo em que seu conteúdo deveria ser assimilado pela pessoa idosa como algo do seu dia a dia, para identificar quais as deficiências e sua extensão no seu dia a dia. Com relação ao componente, Estruturas do corpo, o propósito das perguntas foi avaliar se essa anatomia apresenta deficiências ou não. O terceiro componente, referente a Atividades e Participação, que mensura o desempenho e a capacidade por meio da execução de uma atividade ou ação de situações rotineiras, buscou manter o foco na execução de tal atividade. As perguntas precisavam, nessa parte do instrumento, ter movimento, ou seja, foco prático na vida diária da pessoa idosa, onde ele visualizasse sua rotina diária. Por fim, o quarto componente, Fatores contextuais, que se subdivide em Fatores ambientais e Fatores pessoais. Com relação aos Fatores ambientais, que integram 0 ambiente físico, social e atitudinal em que as pessoas vivem e conduzem o dia a dia, seu objetivo é avaliar se cada item é facilitador ou dificultador, ou seja, barreira, na situação descrita pelo indivíduo. A estrutura seguiu a descrição primeiramente da codificação, igual a apresentada na CIF, a pergunta seguida pelo qualificador, positivo ou negativo.

Como a CIF elenca qualificadores para cada um dos componentes e após essa releitura, entendeu-se que se fazia necessária a inclusão, antes de cada componente da definição dos seus qualificadores, para mensuração da resposta positiva ou negativa de cada categoria. Cada componente possui qualificador ou qualificadores, segundo a própria $\mathrm{CIF}^{5}$.

O instrumento recebeu a descrição do qualificador ou qualificadores de cada componente, antecedendo suas perguntas. Por fim, ao concluir essa estruturação, o IAMPI foi submetido a Validação de conteúdo e aparência, para que o instrumento inicial, pudesse receber os ajustes, considerações, correções e ponderações.

\section{Validação de conteúdo e aparência do Instrumento para a Avaliação Multidimensional da Pessoa Idosa - IAMPI}

Para a realização da validação de conteúdo e de aparência, utilizou-se a técnica de validação de consenso de Carlson ${ }^{12}$, que consiste na obtenção da opinião ou o acordo entre especialistas sobre um determinado fenômeno. Nesta etapa do estudo o grupo de especialistas foi representado por um geriatra, especializado em avaliação multidimensional, de atuação clínica no nível primário e secundário de saúde, por um fisioterapeuta, referência nacional e internacionalmente, no uso da CIF, e o terceiro por uma enfermeira, doutora em Enfermagem e com área de atuação na atenção a pessoa idosa, além da autora principal do estudo, que ficou como líder do grupo. Todos foram escolhidos por conveniência e aceitaram participar dessa etapa, para avaliar o conteúdo e a aparência do instrumento e assinaram o TCLE. Os encontros foram realizados via internet de modo virtual, utilizando o WhatsApp, e-mails e reuniões virtuais, durante o período de dois meses, para avaliar o IAMPI no que diz respeito a validação de conteúdo enfatizando a pertinência, clareza e organização da pergunta em questão, e a validação da aparência, relacionado ao layout, visual e organização geral do instrumento.

As discussões do grupo foram organizadas em aspectos como ajustes, considerações e correções de termos, durante 
os encontros realizados, até a redação das perguntas e a obtenção do consenso da validação de conteúdo e de aparência do instrumento.

\section{RESULTADOS}

Foram identificados na literatura um o total de 184 termos da CIF, dos quais 69 faziam parte das Funções do Corpo, 04 termos ligados a Estruturas do Corpo, 43 termos em Atividades e Participação, 50 termos em Fatores Ambientais e 18 termos com Fatores Pessoais, considerados relevantes pelos especialistas para a avaliação da pessoa idosa. Esses resultados foram utilizados na construção de um segundo instrumento no Google Forms $\AA$, levando em consideração na sua elaboração todos os passos já descritos nesse estudo. O resultado dessa etapa, foi a sinalização de 230 termos da CIF, com IVC $\geq 0.80$, dos quais 96 faziam parte das Funções do Corpo, 09 termos ligados a Estruturas do Corpo, 85 termos em Atividades e Participação, 26 termos em Fatores Ambientais e 14 termos com Fatores Pessoais.

Quando comparado os itens que obtiveram IVC $\geq 0,80$ na segunda fase, pelos profissionais da área de saúde e os termos resultantes da revisão integrativa (RI) com os estudos associados a CIF e a pessoa idosa na literatura da área da saúde, os componentes referentes aos Fatores Pessoais, a RI sinalizou 16 itens e o instrumento da Fase 2, 14 itens, destes 10 itens estavam presentes em ambos. Com relação aos demais componentes, a RI identificou 158 itens e o instrumento Fase 2, 216 itens, dos quais 46 itens estavam presentes em ambas as fases.

Após essa avaliação inicial, foi feito o alinhamento do desenho inicial do instrumento, tendo sempre o foco de um instrumento administrável, de clareza para o avaliador e compreensível para o avaliado.

A 1a Parte do IAMPI recebeu pequenas considerações, com foco maior de sua aparência. Com relação aos Fatores
Pessoais, os itens IVC $\geq 0.80$, em ambas as etapas foram: Ciclo de Vida; Educação; Envelhecimento; Estado civil; Idade; Local residência; Nível socioeconômico; Número de filhos; Ocupação; Problemas orgânicos; Representações sociais e Sexo. Entretanto, os termos Representações sociais e Envelhecimento, também por sugestão dos especialistas, essas categorias permaneceram não incluídas no instrumento (Figura 1).

Foram feitas considerações quanto as informações como Registro Geral (RG) e Cadastro de Pessoa Física (CPF), dados que não precisariam de espaços, da forma como foi colocado no instrumento inicial. Outro ponto discutido e sugerido, foi a não utilização do termo CID-10, apenas CID, já que é uma Classificação que é revisada com certa periodicidade e ocasionaria uma desatualização do instrumento no futuro.

Com relação a informação referente ao Conselho Regional de Medicina - CRM, do(s) médico(s) assistente(s), os especialistas pontuaram a flexibilidade da mesma, e o seu não preenchimento, não impactar na operacionalização do IAMPI. É sabido, que não há, por parte dos indivíduos nem a curiosidade e nem o armazenamento desse tipo de informação, portanto, por ser uma informação que poderá apresentar falta de conhecimento dos avaliados, ela não será obrigatória. Entretanto, caso as possuam, é válida a informação para que possa ser realizado a referência e a contrarreferência das informações obtidas com a aplicação do instrumento. O mesmo se aplica para os Conselhos Federais dos demais profissionais de saúde que fizerem o atendimento à pessoa idosa.

As maiores considerações ocorreram com relação à estruturação da $2^{\underline{a}}$ Parte do IAMPI (Figura 2) e os qualificadores a serem utilizados. Com relação aos ajustes, foram pontuadas as repetições dos conteúdos abordados em cada termo que, apesar de estarem em domínios diferentes, abordavam 
o mesmo teor, por exemplo, o termo b1562 Percepção olfativa (Funções mentais envolvidas na diferenciação de odores) assemelhava-se à b255 - Funções olfativa (Funções sensoriais que permitem sentir odores e aromas).

Figura 1 - Fatores Pessoais e Fatores Ambientais do Instrumento para Avaliação Multidimensional da Pessoa Idosa, João Pessoa, PB, 2021.
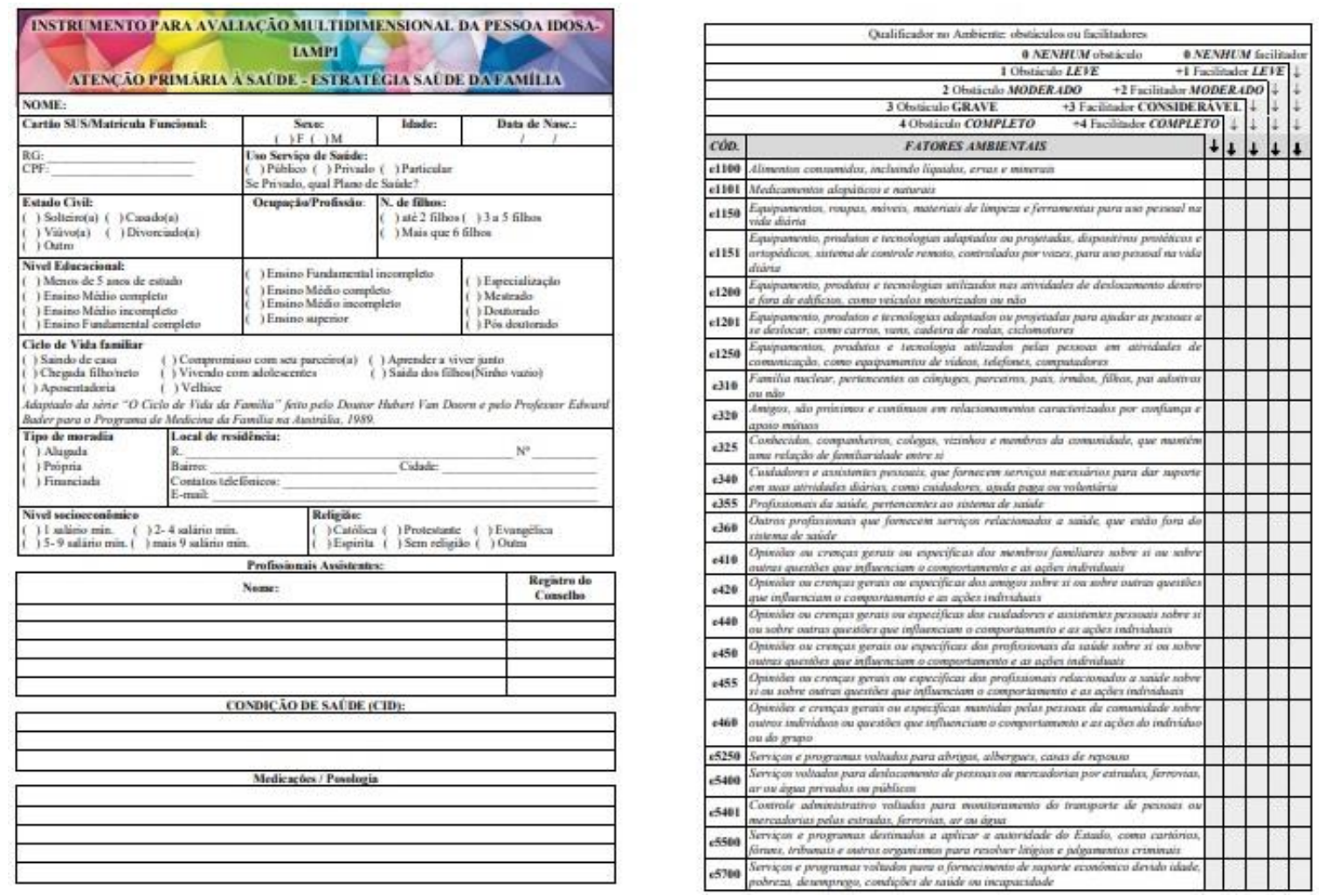

Figura 2 - Fatores Ambientais e Atividades e Participação do Instrumento para Avaliação Multidimensional da Pessoa Idosa, João Pessoa, PB, 2021.
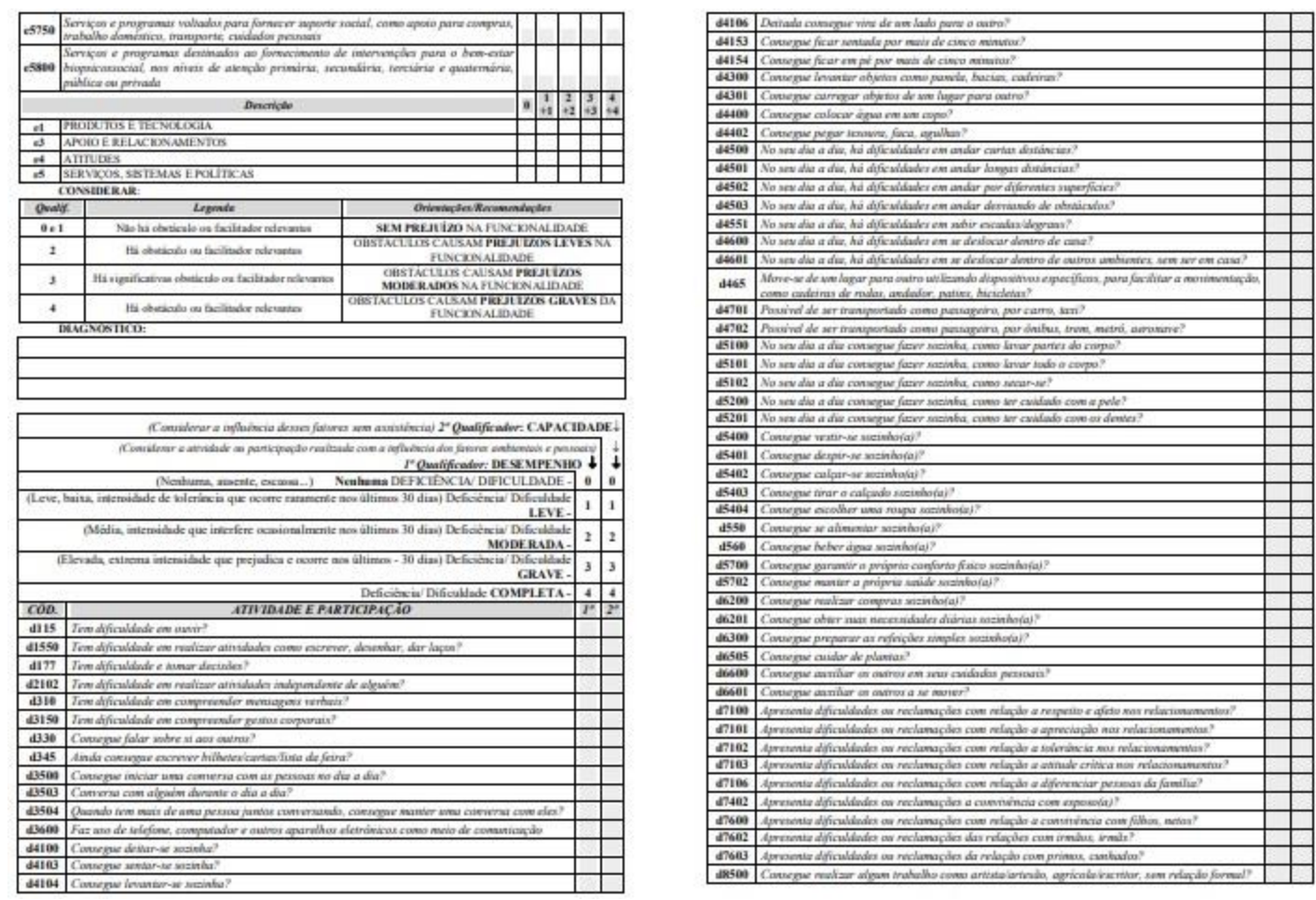
Os ajustes foram feitos a partir dos termos que deveriam permanecer ou deveriam ser extraídos do instrumento, por se repetirem. Observou-se que poucos foram os termos que necessitaram ser extraído da versão inicial, por abordarem o mesmo conteúdo.

Os avaliadores alertaram para 0 entendimento e escrita clara das perguntas, por termo, para que o profissional de saúde que for fazer a avaliação da pessoa idosa, tenha domínio do conteúdo e alcance respostas claras e verídicas, a exemplo, a questão: b7801 Sensação de espasmo muscular, sua pergunta foi descrita com detalhamento de como pode ser sentido um espasmo, ficando: Sensação de contração muscular, involuntário, com ou sem dor (espasmo). Após as considerações dos avaliadores, a melhor redação para que possa ser adquirido conhecimento sobre a sensação de espasmo muscular seria uma redação das possibilidades de como o espasmo pode ser percebido pelo indivíduo, considerou-se que o detalhamento ficou mais claro e abrangente, da forma como foi colocado na versão final.

Outra consideração importantíssima foi com relação à ordem das partes no instrumento, diante do seu objetivo que é a avaliação multidimensional da pessoa idosa, foi sugerido que o instrumento iniciasse com as perguntas referentes ao componente Fatores Ambientais, seguido de Atividades e Participação.

$\mathrm{Na}$ sequência as perguntas referentes a Estruturas do Corpo e por fim Funções do Corpo, apresentado nas figuras 3 e 4 . Consideração essa justificável diante da possibilidade de maior visibilidade e importância dos Fatores Ambientais na avaliação multidimensional da pessoa idosa. Para atender essa sugestão, o IAMPI passou por uma grande alteração na sua aparência, mas sem interferir no conteúdo dos componentes da CIF.

\section{Figura 3 - Atividades e Participação e Estruturas do Corpo do Instrumento para Avaliação Multidimensional da Pessoa Idosa, João Pessoa, PB, 2021.}
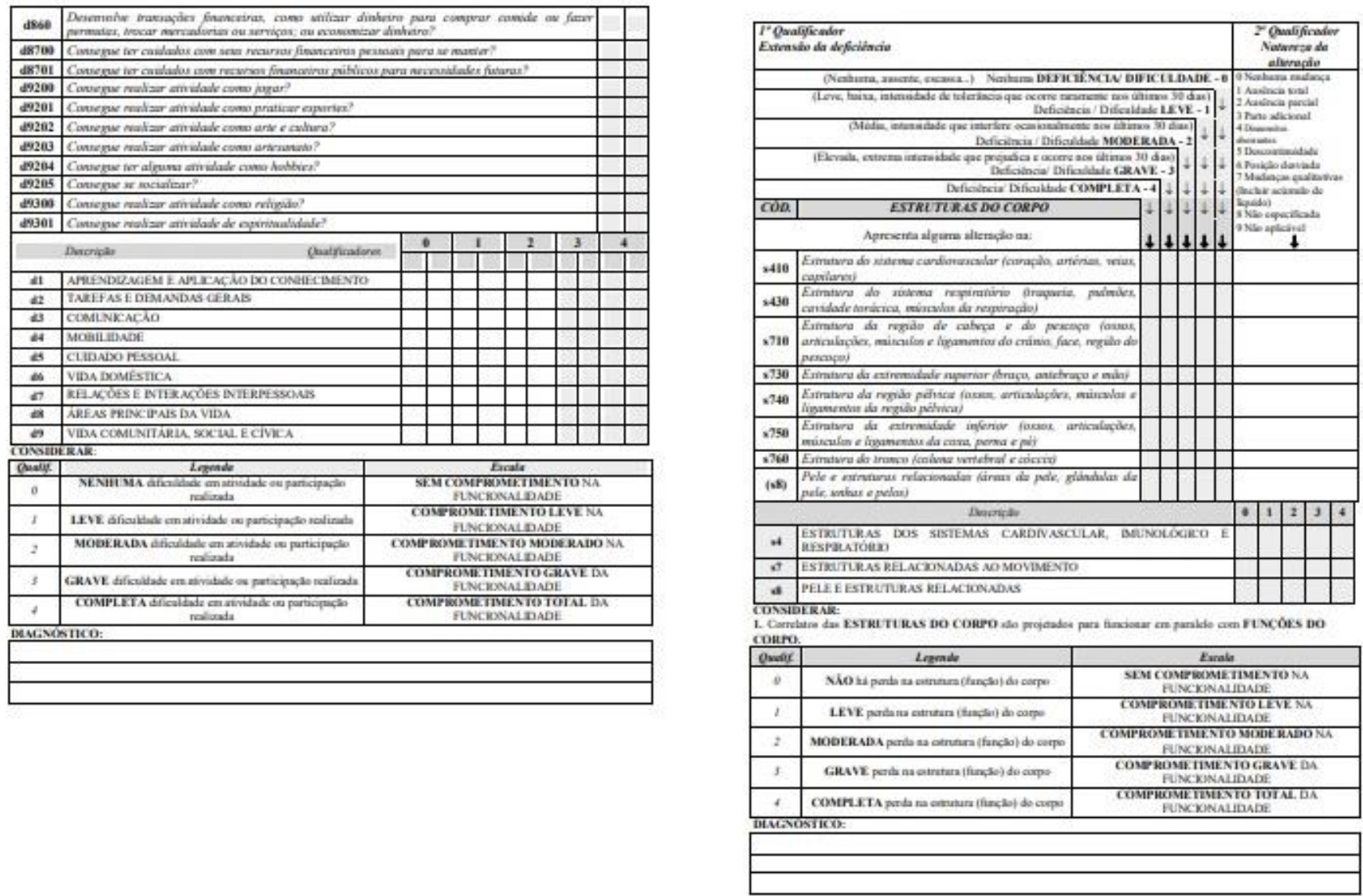
Figura 4 - Funções do Corpo do Instrumento para Avaliação Multidimensional da Pessoa Idosa, João Pessoa, PB, 2021.

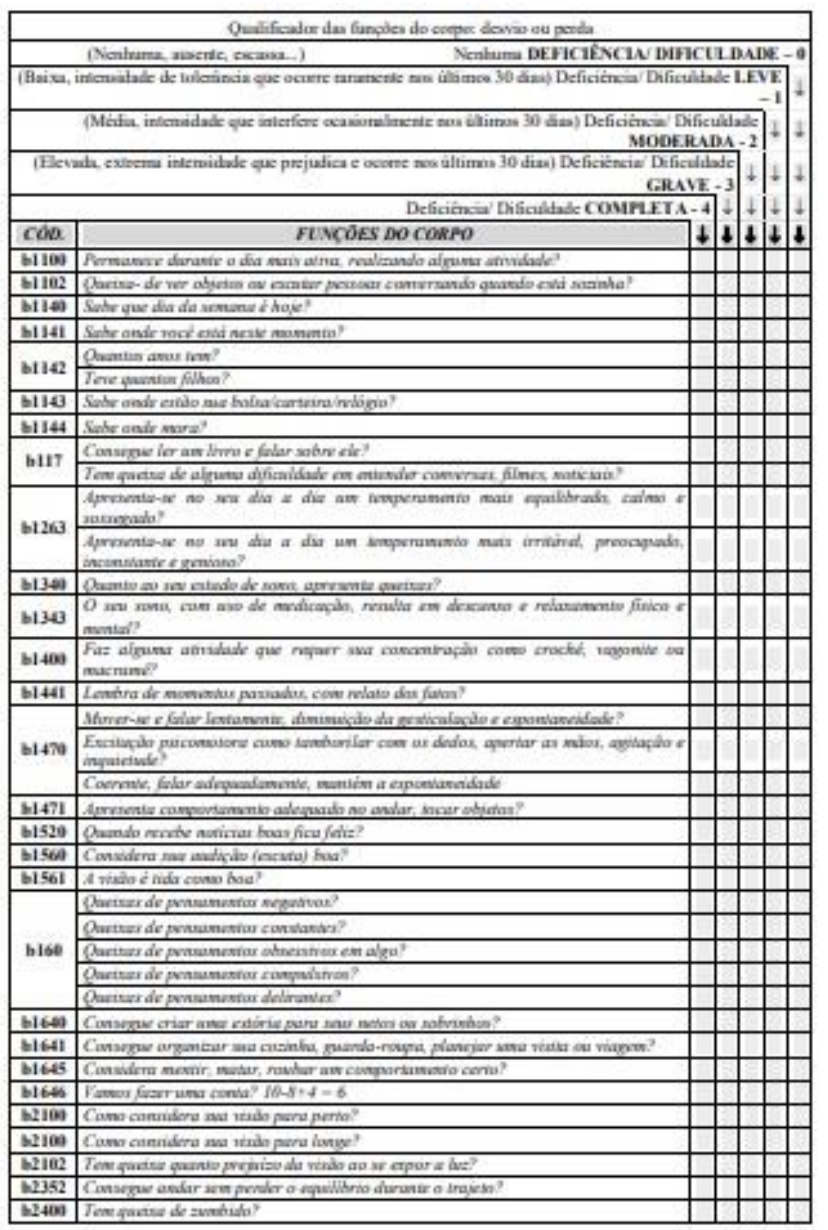

Com relação às correções, foram poucas ocorrências, por exemplo, ajustes de português, repetição das variáveis: Ciclo de Vida e Religião (Fatores Pessoais e dados sociodemográfico). Após todas as correções de sua versão mais atualizada, o Instrumento para Avaliação Multidimensional da Pessoa Idosa precisou ser revisto com relação aos qualificadores da CIF, tendo em vista que, ao longo da validação de conteúdo, foram muito discutidos os qualificadores e a forma como seriam incluídos. Chegou-se ao consenso que seria mais coerente, devido sua compreensão, prática e clareza, o uso de qualificadores genéricos sugerido no estudo de Fontes, Fernandes e Botelho ${ }^{13}$.

A partir das orientações e sugestões pelos especialistas, a inclusão de uma escala

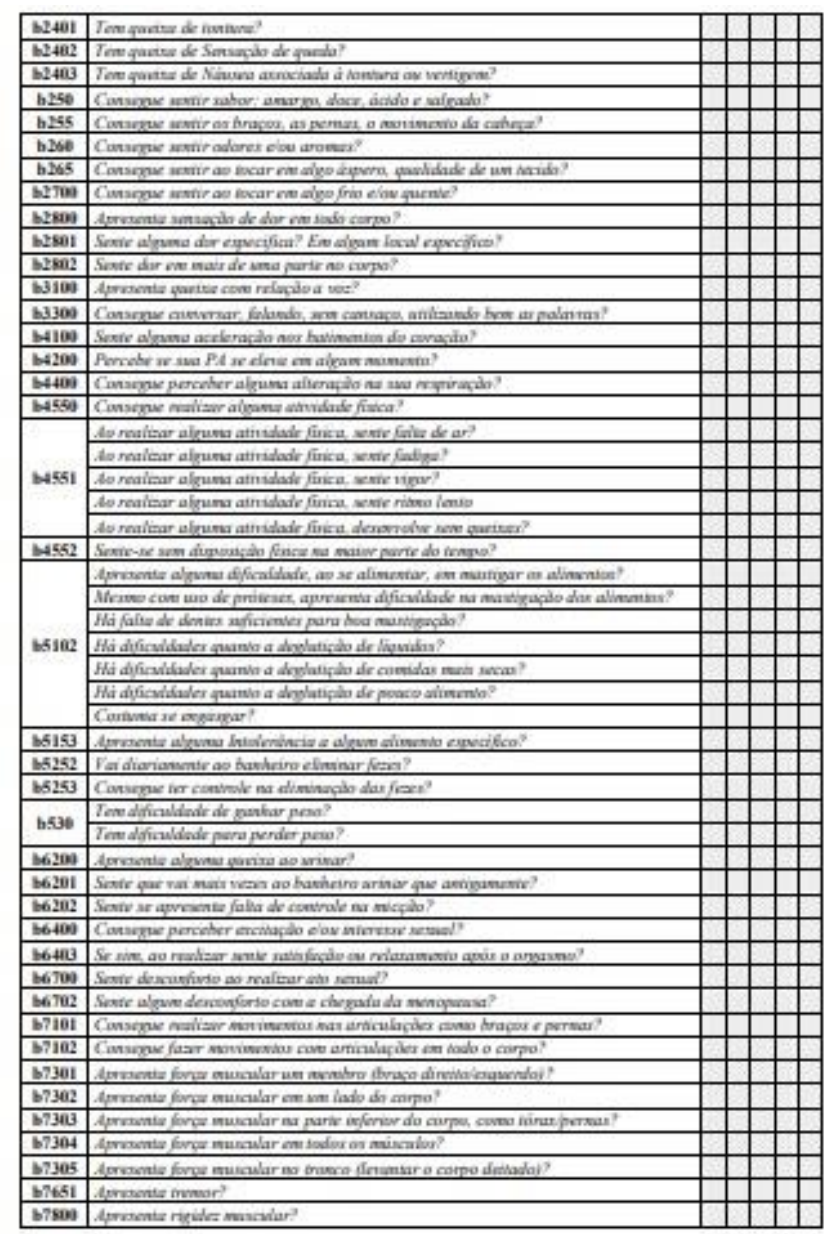

tipo likert com os qualificadores, seria mais prático e daria melhor visibilidade ao avaliador.

Também foi sugerido a inclusão de um espaço para incluir, a partir da avaliação multidimensional da pessoa idosa, o diagnóstico multidimensional em cada um dos componentes do instrumento, para facilitar o desenvolvimento do plano de cuidados da equipe no final do instrumento, conforme pode ser visualizado no layout final do IAMPI, apresentado nas figuras 4 e 5 .

A figura 6 apresenta as metas a curto, médio e longo prazo, que podem ser traçadas após as Atividades e Participação e Estruturas do Corpo. 
Figura 5 - Funções do Corpo e Plano de Cuidado Multidimensional do Instrumento para Avaliação Multidimensional da Pessoa Idosa, João Pessoa, PB, 2021.
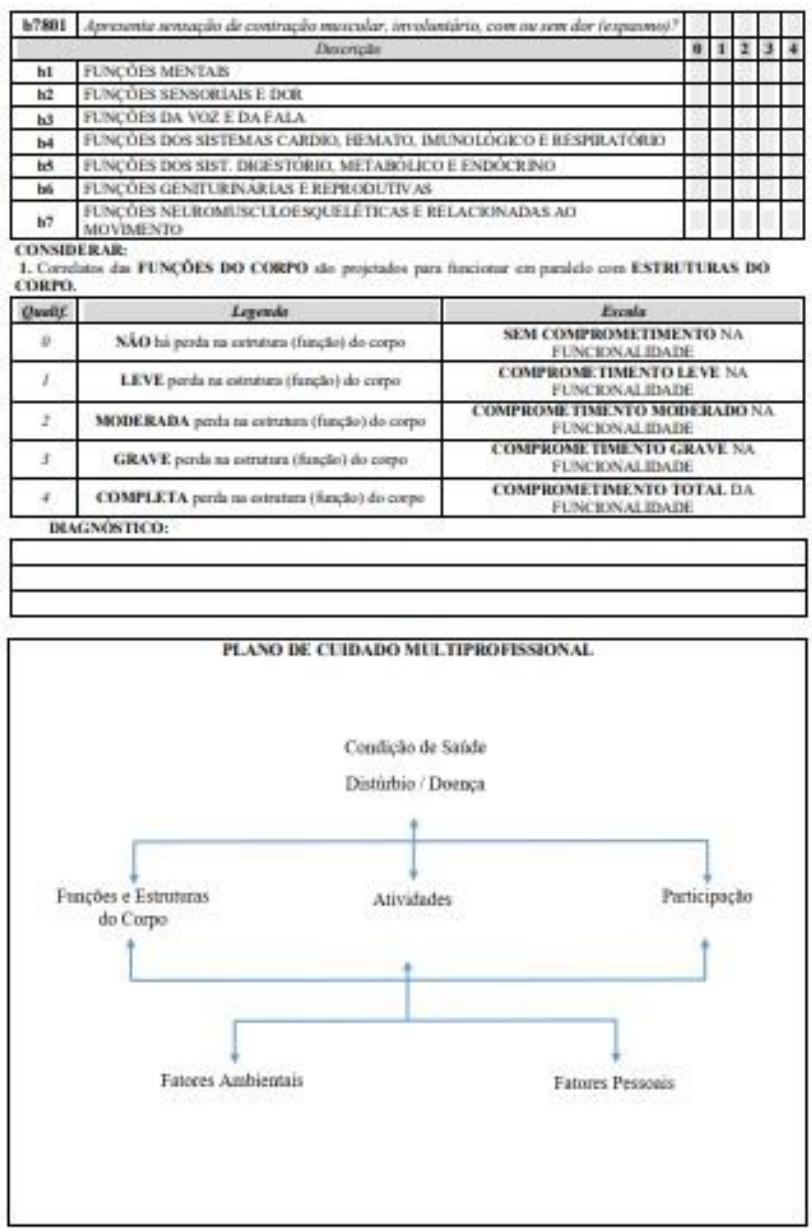

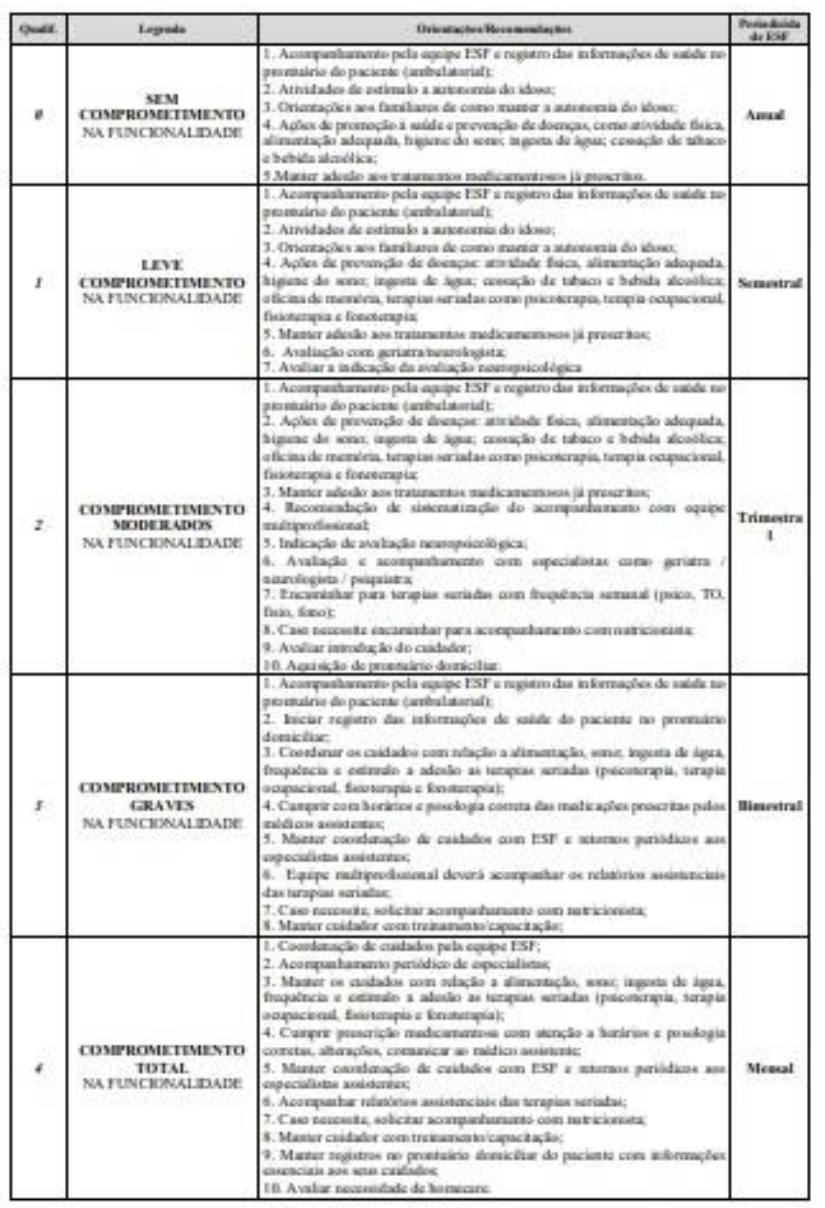

Figura 6 - Campo referente as metas a serem traçados após realização Atividades e Participação e Estruturas do Corpo do Instrumento para Avaliação Multidimensional da Pessoa Idosa, João Pessoa, PB, 2021.

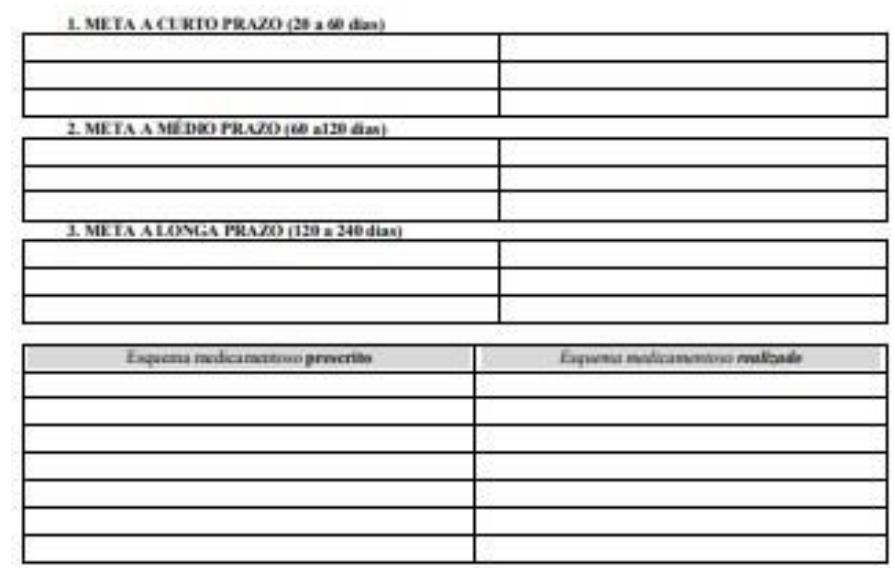

\section{DISCUSSÃO}

O produto final do estudo consiste no Instrumento de Avaliação Multidimensional para a Pessoa Idosa - IAMPI, construído contando com a colaboração de vários

profissionais da saúde, em áreas de atuação distintas e profissões diversas, comprovando o quanto 0 assunto em tese representa interesse e relevância tanto para a prática profissional, quanto para a atuação nos 
diversos níveis de saúde - a academia e pesquisa. Apesar de alguns obstáculos, comuns e recorrentes na pesquisa, a presteza e disponibilidade dos profissionais foram de suma importância tanto para a conclusão das fases metodológicas, como para a finalização do instrumento.

A escolha da CIF foi determinada pelo seu modelo conceitual e sua estrutura teórica, que embasaram o instrumento proposto de avaliação multidimensional da pessoa idosa a ser aplicado por profissionais de saúde, na APS. A CIF é uma classificação abrangente, complexa e ainda de pouca utilização pelos diversos profissionais de saúde ${ }^{14-16}$.

Apesar da CIF ser complexa e de difícil aplicação, o seu modelo proporciona uma linguagem homogênea para todos, traduzida por uma terminologia que abrange especialistas, favorecendo a prática profissional, principalmente na comunicação entre a equipe multiprofissional e profissionais de outras áreas, como também não especialistas e, consequentemente, fortalecendo a prática clínica na saúde, para obtenção de maior resolutividade e sistematização da assistência prestada ${ }^{5}$.

É válido ressaltar que esse instrumento não é um checklist da CIF, nem um core set, ele é um instrumento que possui sua fundamentação teórica em uma classificação universal, com um modelo teórico multidimensional, que embasa toda a proposta desse Instrumento para Avaliação Multidimensional da Pessoa Idosa. Portanto, para facilitar a compreensão dos qualificadores, optou-se por utilizar as palavras deficiência/dificuldades para os componentes Atividade e Participação, Estruturas do Corpo e Funções do Corpo e as palavras obstáculo/facilitador para os Fatores Ambientais.

Sabe-se o quão difícil é a utilização dos qualificadores da $\mathrm{CIF}$, tanto pela sua extensão, quanto pela subjetividade que eles se apresentam. Em leituras e releituras da própria CIF, do Manual de utilização da CIF em Saúde Funcional ${ }^{7}$, alguns artigos ${ }^{13,17-18}$, a possibilidade de utilização de qualificadores genéricos no instrumento, foi sendo desenhada, vislumbrando a possibilidade de se ter o instrumento mais aplicável, em nível primário de saúde.

Comparando os termos identificados entre os momentos da Segunda Fase, de acordo com os domínios da CIF, os termos de maior expressão estão presentes nos domínios Funções do Corpo e Atividades e Participação, que nos mostram o quanto os estudos ainda estão centrados na identificação dos problemas, das perdas, das deficiências, das limitações quanto a execução ou restrição de atividades que o indivíduo poderá enfrentar ${ }^{19}$.

Apesar de já ter sido mencionado, é válido relembrar, que apesar do domínio Fatores Pessoais não constar na CIF, os termos relacionados a esse domínio são sempre referenciados e sinalizados nos estudos pelos profissionais e são as variáveis mais presentes em quase todos os estudos ${ }^{11}$. $\mathrm{Na}$ prática profissional e nas considerações dos estudos apesar de ressaltarem a importância dos fatores ambientais, os avaliadores não fizeram essa sinalização ao serem solicitados.

Diante dos resultados encontrados, constatou-se que os termos em comum nos dois momentos da Segunda fase, foram representativas em todas as partes da CIF, porém é notável que ao apresentar as categorias e seus níveis, o detalhamento das categorias agrega mais especificidade para si, por exemplo, a categoria d920 Recreação e lazer, foi sinalizada na Fase 1 e ao abri seu detalhamento, na Fase 2, permitiu visualizar seus níveis de detalhamento e, consequentemente, obteve relevância nos seguintes termos: d9200 Jogar; d9203 Artesanato; d9204 Hobbies; d9205 Socialização.

Buscando analisar esse resultado a luz da literatura, observa-se que os estudos pontuam a importância da CIF, na atuação 
clínica não só referente ao estudo das deficiências, mas, também na busca de elencar a funcionalidade, enfatizando, como objeto deste estudo, na população idosa ${ }^{19}$.

$\mathrm{Na}$ sua estruturação evidenciou-se o quanto os dados pessoais são importantes e influenciam na saúde das pessoas idosas. Valorizar os dados sócios-demográficos, contendo variáveis como nome, idade, estado civil, sexo, composição familiar, nível educacional e econômico, ciclo de vida e as morbidades referidas, reforça como mais um estudo que identifica a importância dos fatores pessoais e quais os principais fatores pessoais precisam ser colhidos com mais atenção e sistematização ${ }^{11}$.

Entretanto, as informações referentes ao uso de tabaco e uso de substâncias psicoativas, não foram sinalizados em nenhuma das fases pelos avaliadores, portanto não foram incluídos nesse instrumento, mesmo sendo considerados como dados de saúde importantes para avaliação clínica em qualquer faixa etária, além de serem considerados grandes fatores de riscos cardiovascular. Esse é um aspecto que precisa ser considerado quando for feita a validação clínica do IAMPI.

\section{CONSIDERAÇÕES FINAIS}

A proposta inicial da construção de um instrumento que abarcasse componentes de saúde física, mental, funcional, dinâmica familiar, qualidade de vida, qualificadores de saúde, dificultadores da funcionalidade do idoso, foi alcançado e poderá se tornar realidade. Sabe-se o quanto a caminhada foi e será complexa, de muitas dúvidas e considerações, mas que enfrentadas como intervenções para que o IAMPI não seja um instrumento longo, de difícil aplicabilidade e compreensão, mas que tenha seu objetivo claro e bem descrito, facilitando seu manuseio no serviço de saúde ainda na atenção primária à saúde, que deve ser a porta de entrada da pessoa idosa nos serviços de saúde, fortalecendo a prevenção e promoção à saúde, bem como uma base teórica que fundamente a avaliação multidimensional da pessoa idosa.

Ressalta-se a pertinência do modelo teórico selecionado para o estudo - a CIF, uma vez que seu uso pelos profissionais não só geram uma legitimação e sistematização da prática profissional, como também gera informações ricas e reais que facilitam elaborações de políticas públicas mais legítimas e locais, e estatísticas que proporciona melhorias à saúde e ao sistema de saúde como um todo, com apropriação das reais necessidades.

A aplicabilidade desse instrumento, na Atenção Primaria à Saúde, facilitará o reconhecimento dos aspectos multidimensionais que permeiam o dia a dia das pessoas idosas e são imprescindíveis para o serviço de saúde. Ação que acarretará na prestação de uma assistência à saúde sistematizada, pautada no modelo biopsicossocial do indivíduo. Consequentemente, maior resolutividade em suas reais necessidades, podendo acarretar diminuições de encaminhamentos e exposição desnecessárias, qualificando assim a escuta e os desdobramentos mais eficazes e com possibilidade de coordenação de cuidados por qualquer membro da equipe multiprofissional.

Acredita-se que a grande implicação do estudo é transformar a CIF em uma Classificação de Funcionalidade, com foco na saúde e ter seu uso igualitário entre as demais classificações e nas diversas profissões, fazer presente seu modelo biopsicossocial de conhecimento e parte integrante na prática da clínica ampliada, para um empoderamento baseado em evidências para um melhor plano de cuidado da pessoa idosa. 


\section{REFERÊNCIAS}

1.Duarte YAO, Andrade C L, Lebrão M L. O índex de Katz na avaliação da funcionalidade dos idosos. Revista da Escola de Enfermagem da USP [online]. 2007, vol. 41, no. 2 [Acessado 10 Abril 2020], p. 317-325. Disponível em: <https://doi.org/10.1590/S0080-62342007000200021>. Epub 28 Ago 2007. ISSN 1980-220X. https://doi.org/10.1590/S0080-62342007000200021

2.Santos GS, Cunha ICKO. Capacidade funcional e sua mensuração em idosos: uma revisão integrativa. REFACS [online]. 2014, vol. 2, no. 3, p. 268-78.

3. Moraes EN. Atenção à saúde do idoso: aspectos conceituais. Brasília: Organização PanAmericana da Saúde; 2012.

4. Sposito G, D'Elboux MJ, Neri AL, Guariento ME. Satisfaction with life and functionality among elderly patients in a geriatric outpatient clinic. Ciênc. saúde coletiva [online], 2013, vol. 18, no. 12, p. 3475-82. DOI: http://dx.doi.org/10.1590/S1413-81232013001200004.

5. Organização Mundial de Saúde. CIF: Classificação Internacional de Funcionalidade, Incapacidade e Saúde [Centro Colaborador da Organização Mundial da Saúde para a Família de Classificações Internacionais, org.]. 1. ed,. 2. reimpr. Atual. São Paulo: EDUSP; 2015. 333p.

6. Araújo ES. CIF: Uma discussão sobre linearidade no modelo biopsicossocial. Revist Fisioterapia em Saúde Funcional. 2013, vol. 2, no. 1, p. 6-13.

7. Araújo ES. Manual de utilização da CIF em saúde funcional. São Paulo: Andreoli; 2011.

8. Polit DF, Beck CT. Fundamentos da pesquisa em enfermagem: avaliação de evidências para a prática de enfermagem. 7. ed. Porto Alegre: Artmed, 2011.

9. Conselho Nacional de Saúde (BR). Resolução 466, de 12 de dezembro de 2012. Diretrizes e normas regulamentadoras de pesquisa envolvendo seres humanos: Brasília, 2012.

10. Alexandre NMC, Coluci MZO. Validade de conteúdo nos processos de construção e adaptação de instrumentos de medidas. Ciênc saúde coletiva, Rio de Janeiro [online]. vol.16, no.7, p. 30613068, Jul 2011. ISSN 1413-8123. DOI:10.1590/S1413-81232011000800006.

11. Medeiros EM, Beserra PJF, Nóbrega MML. Instrumentos utilizados na avaliação da pessoa idosa: revisão integrativa nos últimos dez anos. International Journal of Development Research. vol. 11, 2021. DOI: /10.37118/ijdr.20873.01.2021.

12. Carlson J. [Consensus validation process: a standardized research method to identify and link the relevant NANDA, NIC and NOC terms for local populations]. In Paper the International Journal of Nursing Terminologies and Classifications. vol. 17, no. 1, p. 23-4, 2006.

13. Fontes AP, Fernandes AA, Botelho MA. Funcionalidade e incapacidade: aspectos conceptuais, estruturais e de aplicação da Classificação Internacional de Funcionalidade, Incapacidade e Saúde (CIF). Rev Port Saúde Pública, vol. 28, no. 2, p. 171-8. 2010. 
14. Araújo ES, Buchalla CM. [The use of the International Classification of Functioning. Disability and Health in health surveys: a reflexion on its limits and possibilities]. Rev Bras Epidemiol, vol. 18, no.3, p. 720-4, 2015.

15.Araújo ES. 2016. CIF-WIN-S 2017. Revista Científica CIF Brasil. Vol. 6, no. 6, p. 2-5.

16. Castro SS, Castaneda L, Araujo ES, Buchalla CM. [Functioning assessment in Brazilian health surveys: discussions about International Classification of Functioning, Disability and Health-based tools]. Rev Bras Epidemiol. 2016;19(3):679-687. DOI: https://doi.org/10.1590/1980$\underline{5497201600030018 .}$.

17. Maia LC, Colares TF, Moraes EN, Costa SM, Caldeira AP. Idosos robustos na atenção primária: fatores associados ao envelhecimento bem-sucedido. Rev Saúde Pública, vol.54, no. 35, 2020. DOI: http:/doi.org/10.11606/s1518-8787.2020054001735.

18. Piexak DR, Cezar-Vaz MR, Bonow CA. [International Classification of Functioning, Disability and Health: a Content Analysis]. Revista de Pesquisa Cuidado é Fundamental Online, [S.I.], vol. 11, no. 2, p. 363-369, jan. 2019. DOI: http://dx.doi.org/10.9789/2175-5361.2019.v11i2.363-369.

19. Medeiros EM, Beserra PJF, Medeiros ACT, Medeiros FAL, Nóbrega MML. [Categories of the International Classification of Functionality, Disability and Health In Studies with the elderly: integrative review]. In: 6 th International Symposium: ICF Education, 2020, Ribeirão Preto - SP (Online). BOOKLET The 6 th International Symposium: ICF Education. São Paulo - SP: CIFBrasil, 2020. vol. 1. p. 7-7. 